\title{
Optimal Offloading of Kubernetes Pods in Three-Tier Networks
}

This paper was downloaded from TechRxiv (https://www.techrxiv.org).

\section{LICENSE}

CC BY-NC-SA 4.0

SUBMISSION DATE / POSTED DATE

14-02-2022 / 22-02-2022

\section{CITATION}

Carmona, Estela; ladanza, Francesco; Siddiqui, Muhammad Shuaib (2022): Optimal Offloading of Kubernetes Pods in Three-Tier Networks. TechRxiv. Preprint. https://doi.org/10.36227/techrxiv.19169021.v1

DOI 


\title{
Optimal Offloading of Kubernetes Pods in Three-Tier Networks
}

\author{
Estela Carmona-Cejudo*, Francesco Iadanza ${ }^{\dagger}$, Muhammad Shuaib Siddiqui* $^{*}$ \\ ${ }^{*}$ Software Networks, i2CAT Foundation, Barcelona, Spain \\ ${ }^{\dagger}$ Research and Development Laboratory, Engineering Ingegneria Informatica S.p.A., Rome, Italy \\ Email: *\{estela.carmona, shuaib.siddiqui\}@i2cat.net, ${ }^{\dagger}$ francesco.iadanza@eng.it
}

\begin{abstract}
By pushing resources to far-edge servers located in the proximity of users, edge computing can greatly reduce endto-end transmission delays. Task offloading in multi-tier networks refers to the optimization of which tasks should be offloaded from the far-edge to the edge and the cloud. Moreover, the containerization of applications can further reduce resource and time consumption and, in turn, the latency of such applications. Even though Kubernetes has become the de facto container orchestrator, not many works have considered the offloading of containerized applications in Kubernetes clusters spanning from cloud to faredge. In this work, the problem of offloading Kubernetes tasks (or pods) in three-tier networks is formulated and optimized. First, a utility function is presented in terms of the cumulative weighted pod response time, and a utility minimization problem with central processing unit (CPU) constraints is presented. Based on the optimal theoretical solution to this problem, a three-tier offloading decision algorithm (TTODA) is developed. Vertical scaling is considered, and specific hardware capabilities of each node are taken into account by setting specific SLAs that are fed back to the algorithm. Numerical results show that TTODA outperforms a typical Kubernetes QoS model based on first-in, first-served algorithm (FIFSA) in terms of utility, average pod response time, and usage of far-edge CPU. Further, TTODA achieves an excellent trade-off between performance and computational complexity, and thus it can help achieve the requirements of latency-sensitive applications. Moreover, TTODA can easily be extended to scenarios with joint memory and CPU constraints.
\end{abstract}

\section{INTRODUCTION}

The use of containers to virtualize applications has recently become popular, since it offers a more lightweight and less resource and time consuming solution than virtual machines, thus reducing the computational cost of delay-sensitive applications and, in turn, their execution delay. Docker and Kubernetes have become the most popular tools for container orchestration [1]. The smallest computing units that can be deployed in

This paper is the authors' pre-print version of the following work: E. Carmona-Cejudo, F. Iadanza M. S. Siddiqui, "Optimal Offloading of Kubernetes Pods in Three-Tier Networks," 2022 IEEE Wireless Communication and Networking Conference Workshops (WCNC Wkshps).

This document has been provided by the contributing authors as a means to ensure timely dissemination of scholarly and technical work on a noncommercial basis. Copyright and all rights therein are maintained by the authors or by other copyright holders, not withstanding that they have offered their works here electronically. It is understood that all persons copying this information will adhere to the terms and constraints invoked by the corresponding copyright. This work may not be reposted without the explicit permission of the copyright holder.
Kubernetes are known as pods, i.e. groups of application containers, with shared storage and network resources [2].

Moreover, the requirements of latency-sensitive applications can be difficult to meet in traditional cloud-centric networks, even when applications are containerized. In this sense, edge computing can help achieve ever-growing computational demands in fifth generation (5G) networks, by bringing computing capabilities to the far-edge of the network [3]. By pushing resources to far-edge servers in the proximity of users, edge computing can greatly reduce end-to-end latency. However, computational resources at the far-edge are normally smaller than at the edge and cloud tiers. In settings with large numbers of users or computational demanding applications, far-edge resources are unlikely to be enough to meet the requirements of all pods, and it is necessary to offload some pods to the edge or the cloud. A thorough survey and taxonomy on existing task offloading research in two-tier networks was carried our in [3], and several challenges that have not yet been properly addressed in the literature were found. For example, some previous works only considered the average response time of all tasks [4] or the long-term average response of each task [5], which could lead to service level agreement (SLA) violations in practice. The work in [6] considered both horizontal and vertical scaling, and proposed an algorithm to minimize task duration in three-tier cooperative networks. In [7], the authors proposed a service caching and offloading framework with the objective of reducing the overall system delay and energy consumption, but did not consider the time delay achieved by individual tasks. A heuristic algorithm for the offloading of V2X tasks in cooperative three-tier networks was proposed in [8], and it was proved that network delays are significantly reduced with respect to two-tier networks.

Even though Kubernetes has become the de facto container orchestrator, not many works [9], [10] have studied the offloading of containerized applications in Kubernetes clusters spanning from cloud to far-edge. Kubernetes presents some features that differentiate it from other popular productiongrade container orchestration tools. While Docker [11] runs containers on single host machines, Kubernetes applications can be split up into a set of pods and run over several distributed nodes. Self-healing, load balancing and resource management are supported through different quality of service (QoS) models based on resource limits such as central processing unit (CPU) 
and memory. Kubernetes also supports horizontal auto-scaling, such that the response to varying loads is to scale the number pods up or down. Further, Kubernetes offers unified application programming interfaces (APIs) for external management. However, Kubernetes QoS models do not account for the hardware diversity that is typical of edge and far-edge settings; these QoS models assume elastic resource availability across all nodes, and rely on resource limits that can be changed only through auto-scaling. Thus, it is necessary to design new QoS models that take into consideration specific hardware capabilities and SLAs across all network tiers.

The contributions of this paper are as follows. Differently to other works, the use of Kubernetes as a container orchestrator in three-tier networks is considered, and the granularity of applications to be offloaded is defined in terms of Kubernetes pods. Vertical scaling is considered, and specific hardware capabilities of each node are taken into account by setting specific SLAs that are fed back to the algorithm. An utility function is formulated and a theoretical optimization framework is proposed, where the sum of the weighted pod response time is minimized under CPU constraints, and applications are prioritized according to their expected QoS. The optimization problem is solved by applying the Lagrange dual function method [12], and a three-tier offloading decision algorithm (TTODA) is presented. Numerical simulations demonstrate that TTODA outperforms a typical Kubernetes QoS model based on first-in, first-served algorithm (FIFSA) in terms of utility, average pod response time, and usage of far-edge resources. Moreover, TTODA yields an excellent trade-off between performance and implementation complexity, and therefore it can aid meet the requirements of delay-sensitive applications.

The rest of this paper is organized as follows. Section II presents the system model under consideration. The optimization problem of interest is formulated in Section III. Section V presents the proposed TTODA. Numerical results are given in Section VI. Finally, Section VII concludes the paper.

\section{System Model}

The system arcihtecture considered in this work is equivalent to that being developed under the H2020 Pledger project [13], as part of a use case for enhancing the safety of vulnerable road users in the city of Barcelona, Spain. It consists of a Kubernetes-based cluster comprising a cloud, an edge node and a far-edge node, as represented in Fig. 1. All nodes are connected through fiber optic links, which introduce network delays. OpenStack [14] is employed as the virtual infrastructure manager. Three OpenStack compute nodes are set-up in the cloud tier environment, and another one in the edge tier. Kubernetes runs on all cloud and edge nodes, as well as on a bare-metal far-edge server. One node in the cloud serves as the Kubernetes master node, and the remaining cloud, edge and far-edge nodes serve as Kubernetes worker nodes.

It is assumed that the offloading of tasks can take place between the far-edge and the edge or the cloud tiers, depending on the resource usage at each tier and a pod weight factor,

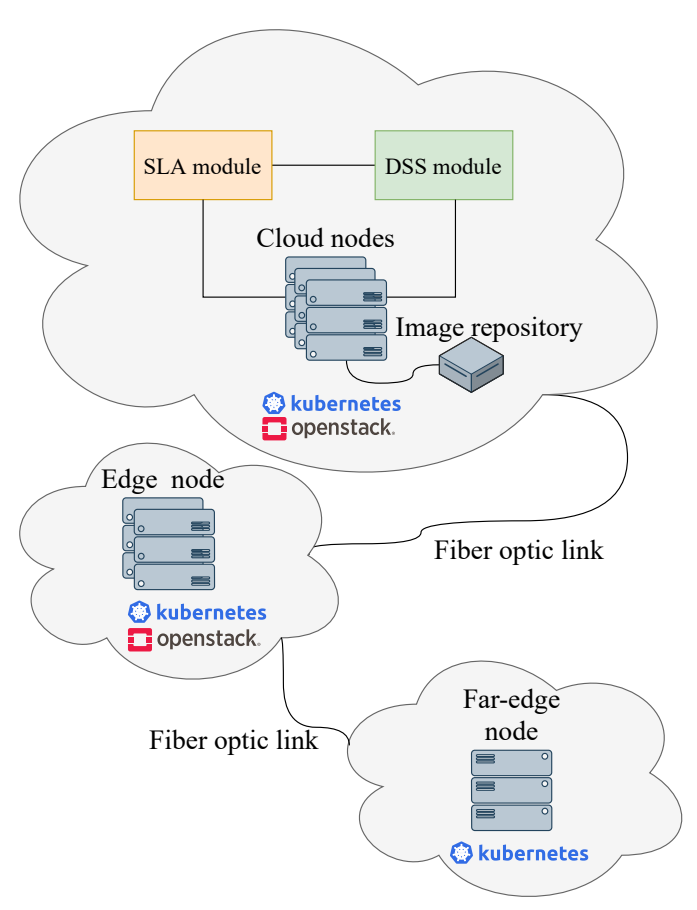

Fig. 1. Architecture setup.

which models the execution priority and resource usage of any given pod. It is also assumed that the edge and far-edge nodes are CPU constrained, but cloud resources are always large enough to absorb the workload of offloaded pods, i.e. the cloud tier is not resource constrained. It is also assumed that all CPU values are semantically identical, i.e. the CPU performance of all nodes is constant for a fixed computational load.

All orchestration and offloading decision tasks are performed by a set of centralized components that are aware of the node distribution, the specific applications running on each node at any given time, and the resource occupancy of nodes [15]. Specifically, offloading decisions based on an offloading algorithm are performed by a central decision support system module (DSSM). The service level agreement module (SLAM) monitors the resource usage of all nodes through a monitoring agent running in the Kubernetes cluster, and the DSSM dynamically adjusts application resource limits based on data fedback from the SLAM. The output of the DSSM is connected to a service orchestrator that directly communicates with the Kubernetes master node through its API, and it requests the offloading of pods as required. For simplicity, it is assumed that the actions performed by the SLAM, DSSM, orchestrator and Kubernetes master node are instantaneous.

Note that Kubernetes supports two types of pod resource quotas: request quotas represent minimum guaranteed resource assignment per pod, whereas limit quotas define the largest resource assignment that can be allocated to a pod. Both types of resource quotas are considered in this work: as the computational load increases, more resources are assigned to 
each pod, until the limit quota is achieved. If the load increases further, additional pods are deployed, and the load balancing is automatically applied among them.

In our system model, a total of $N$ pods compete for resources simultaneously during any given time instant. Several replicas of a pod may be running simultaneously, and each one of the replicas is considered as an independent pod. All applications are assumed to be stateless, with no logic dependencies among pods. Available CPU resources at the far edge and edge servers during a given time instant are represented by $\mathcal{R}_{f}$ and $\mathcal{R}_{e}$, respectively. Pod $n$ requests a certain amount of CPU, denoted as $R_{n}$. In the event that $\sum_{n=1}^{N} R_{n}>\mathcal{R}_{f}$, the far edge server does not have enough CPU resources to handle all pods' requirements, and it is necessary to offload some pods to the edge. Further, if $\sum_{n=1}^{N} R_{n}>\mathcal{R}_{f}+\mathcal{R}_{e}$, the far edge and edge servers together are unable to handle all pods' requirements, and it is necessary to offload some pods to the cloud. It is assumed that pod images are always pre-loaded in the far edge node. At the edge and cloud servers, pod images must be downloaded first from a local image repository, unless another equal pod has previously been instantiated.

\section{PRoblem Formulation}

The problem formulation presented in this work seeks to optimize the offloading of Kubernetes pods in order to minimize the cumulative pod response time, weighted by the priority levels and resource usage requirements of pods. Only CPU constraints are considered, although the formulation below can be extended in a straightforward manner to consider additional constraints such as memory [10].

Variable $\delta_{n}$ models pod $n$ 's total response time (node-to-node latency, instantiation time and pre-loading time) as

$$
\delta_{n}=x_{n}\left(l_{n, e}+p_{n, e}\right)+y_{n}\left(l_{n, c}+p_{n, c}\right)+i_{n},
$$

where $l_{n, e}$ is the average latency from the far-edge node to the edge node, and $l_{n, c}$ is the average latency from the far-edge node to the cloud. $i_{n}$ represents the instantiation time of pod $n$. $p_{n, e}$ and $p_{n, c}$ are the pre-loading times required at the edge and cloud nodes, respectively. For simplicity, the execution time of pods is ignored in the definition of $\delta_{n}$. It is assumed that all pod images are already pre-loaded in the far-edge node; however, only images of pods that have previously been offloaded to the cloud and edge servers are pre-loaded there. Further, $x_{n}$ and $y_{n}$ are binary variables, respectively defined as

$$
\begin{aligned}
& x_{n}= \begin{cases}1, & \text { if pod } n \text { is offloaded to the edge, } \\
0, & \text { otherwise }\end{cases} \\
& y_{n}= \begin{cases}1, & \text { if pod } n \text { is offloaded to the cloud, } \\
0, & \text { otherwise }\end{cases}
\end{aligned}
$$

Delay-sensitive applications can be classified according to their latency and response time requirements, and their execution priority [16]. Thus, the utility function to be optimized in this work is the weighted sum of pod reponse times, given by

$$
U\left(x_{n}, y_{n}\right)=\sum_{n} w_{n} \delta_{n},
$$

with $w_{n}$ the weight factor, defined as $w_{n}=T_{n} / \omega_{n}$, where $\omega_{n}$ is a discrete priority value; a larger value of $\omega_{n}$ indicates higher execution priority. $T_{n}$ is the CPU usage of pod $n$, normalized with respect available resources at the far-edge and edge nodes.

The problem formulation considered in this work is given by

$$
\begin{array}{ll}
\min _{x_{n}, y_{n}} & \sum_{n} w_{n} \delta_{n} \\
\text { s.t. } & x_{n} \in\{0,1\}, \forall n, \\
& y_{n} \in\{0,1\}, \forall n, \\
& x_{n} \neq y_{n}, \forall n, \\
& \sum_{n}\left(1-x_{n}-y_{n}\right) R_{n} \leq \mathcal{R}_{f}, \forall n, \\
& \sum_{n} x_{n} R_{n} \leq \mathcal{R}_{e}, \forall n
\end{array}
$$

Constraints (5b), (5c) and (5d) indicate that pod $n$ can only be deployed in one location, either in the far-edge, in the edge or in the cloud. Constraint (5e) ensures that the sum of required $\mathrm{CPU}$ resources of pods running in the far edge is lower than or equal to available CPU resources at the far edge server. Constraint (5f) ensures that the sum of required CPU resources of pods running in the edge is lower than or equal to available CPU resources at the edge server.

In order to simplify the analysis, constraints (5e) and (5f) can be converted into equivalent dimensionless units, i.e. $\hat{R}_{n}=R_{n} / \mathcal{R}_{f}$ and $\tilde{R}_{n}=R_{n} / \mathcal{R}_{e}$, respectively, where $0 \leq$ $\left\{\hat{R}_{n}, \tilde{R}_{n}\right\} \leq 1$. Therefore, constraints (5e) and (5f) can be respectively written as

$$
\begin{aligned}
& \sum_{n}\left(1-x_{n}-y_{n}\right) \hat{R}_{n} \leq 1, \\
& \sum_{n} x_{n} \tilde{R}_{n} \leq 1 .
\end{aligned}
$$

\section{Two-STEP OfFlOADING OptimizATION}

The optimal solution to problem (5) can be used to develop an optimal offloading algorithm. Such solution is derived below, and then applied in Section V to develop a three-tier offloading decision algorithm (TTODA). Such algorithm can be applied to dynamically perform offloading in three-tier networks where resources allocated to pods as well as pod instances can be scaled up or down.

First, problem (5) is reformulated such that each constraint function only depends on one variable. The equivalent problem is solved by minimizing the objective function over one variable first, and then minimizing over the remaining variable.

Assume $z_{n}=x_{n}+y_{n}$, where

$$
z_{n}= \begin{cases}1, & \text { if pod } n \text { is offloaded to the edge or cloud, } \\ 0, & \text { otherwise. }\end{cases}
$$

I.e. $z_{n}$ denotes whether pod $n$ is offloaded or not, independently of whether it is allocated to the edge node or to the cloud.

Further, the binary nature of variables $x_{n}, y_{n}$ and $z_{n}$ adds complexity to the problem in (5). In order to simplify the 
analysis, variables $x_{n}, y_{n}$ and $z_{n}$ are relaxed and transformed into equivalent real versions, $\left\{\hat{x}_{n}, \hat{y}_{n}, \hat{z}_{n}\right\} \in \mathbb{R}$. Problem (5) is then reformulated in standard form as

$$
\begin{array}{ll}
\min _{\hat{x}_{n}, \hat{z}_{n}} & \sum_{n} w_{n} \hat{\delta}_{n} \\
\text { s.t. } & \sum_{n}\left(1-\hat{z}_{n}\right) \hat{R}_{n}-1 \leq 0, \forall n, \\
& \sum_{n} \hat{x}_{n} \tilde{R}_{n}-1 \leq 0, \forall n
\end{array}
$$

where $\hat{\delta}_{n}=\hat{x}_{n}\left(l_{n, e}+p_{n, e}\right)+\left(\hat{z}_{n}-\hat{x}_{n}\right)\left(l_{n, c}+p_{n, c}\right)+i_{n}$.

According to [12], it is possible to minimize the objective function $U$ in problem (9) by first minimizing over one of the variables, and then minimizing over the other one. In the analysis below, problem (9) is first minimized over $\hat{z}_{n}$, and then it is minimized over $\hat{x}_{n}$.

Problem (9) is solved by applying the Lagrange dual function method [12]. When minimizing over $z_{n}$, the dual function of (9) can be written as

$$
\begin{array}{r}
g=\inf _{\hat{z}_{n}}\left\{\left(w_{n}\left(l_{n, c}+p_{n, c}\right)-\lambda \hat{R}_{n}\right) \hat{z}_{n}+w_{n} i_{n}+\right. \\
\left.w_{n}\left(l_{n, e}+p_{n, e}-l_{n, c}-p_{n, c}\right) \hat{x}_{n}+\lambda\left(\hat{R}_{n}-1\right)\right\},
\end{array}
$$

where inf denotes infimum. After some algebraic manipulations, the nontrivial lower bound to problem (9) is found as

$$
\begin{aligned}
\hat{z}_{n}=w_{n} i_{n}+w_{n}\left(l_{n, e}+p_{n, e}-\right. & \left.l_{n, c}-p_{n, c}\right) \hat{x}_{n} \\
& +\lambda\left(\hat{R}_{n}-1\right),
\end{aligned}
$$

and

$$
\begin{aligned}
\tilde{U}\left(\hat{x}_{n}\right) & =\min _{\hat{z}_{n}} U\left(\hat{x}_{n}, \hat{z}_{n}\right)=\sum_{n} w_{n}\left(i_{n}+\left(l_{n, e}+p_{n, e}\right.\right. \\
& \left.\left.-l_{n, c}-p_{n, c}\right) \hat{x}_{n}+\left(l_{n, c}+p_{n, c}\right)\left(1-\frac{1}{\hat{R}_{n}}\right)\right) .
\end{aligned}
$$

The problem in (9) is then equivalent to

$$
\begin{array}{ll}
\min _{\hat{x}_{n}} & \tilde{U}\left(\hat{x}_{n}\right) \\
\text { s.t. } & \sum_{n} \hat{x}_{n} \tilde{R}_{n}-1 \leq 0, \forall n .
\end{array}
$$

The dual function of problem (13) is

$$
\begin{gathered}
\tilde{g}=\inf _{\hat{x}_{n}}\left\{w_{n}\left(l_{n, e}+p_{n, e}-l_{n, c}-p_{n, c}+\tilde{\lambda}\right) \hat{x}_{n}\right. \\
\left.+w_{n} i_{n}+\left(l_{n, c}+p_{n, c}\right)\left(1-\frac{1}{\hat{R}_{n}}\right)-\tilde{\lambda} \tilde{R}_{n}\right\} .
\end{gathered}
$$

After finding the nontrivial lower bound to problem (13) and performing some algebraic manipulations, the optimal solution to problem (9) is given by

$$
\begin{aligned}
\hat{x}_{n}= & w_{n} i_{n}+\left(l_{n, c}+p_{n, c}\right)\left(1-\frac{1}{\hat{R}_{n}}\right) \\
& -\left(l_{n, c}+p_{n, c}-l_{n, e}-p_{n, e}\right) \tilde{R}_{n} .
\end{aligned}
$$

Further, $\hat{z}_{n}$ can be found by substituting (15) into (11), and $\hat{y}_{n}$ is given by $\hat{y}_{n}=\hat{z}_{n}-\hat{x}_{n}$. Assuming

$$
\begin{aligned}
& \mathcal{X}=\left[\hat{x}_{1}, \cdots, \hat{x}_{n}, \cdots, \hat{x}_{N}\right], \\
& \mathcal{Y}=\left[\hat{y}_{1}, \cdots, \hat{y}_{n}, \cdots, \hat{y}_{N}\right],
\end{aligned}
$$

the values in $\mathcal{X}$ and $\mathcal{Y}$ that minimize the utility function $U$ are $\min \mathcal{X}$ and $\min \mathcal{Y}$, respectively. Thus, it is optimal to schedule the pod that yields $\min \mathcal{X}$ to the far-edge, and the pod that yields $\min \mathcal{Y}$ to the cloud. By definition, $\min \mathcal{X}$ and $\min \mathcal{Y}$ are disjoint sets where $x_{n} \neq y_{n}, \forall n$. Therefore, the optimal solution to the original problem in (5) is given by

$$
\begin{aligned}
& x_{n}= \begin{cases}0, & \text { for } n \text { such that } \hat{x}_{n}=\min \mathcal{X}, \\
1, & \text { otherwise }\end{cases} \\
& y_{n}= \begin{cases}0, & \text { for } n \text { such that } \hat{y}_{n}=\min \mathcal{Y}, \\
1, & \text { otherwise }\end{cases}
\end{aligned}
$$

\section{THREE-TIER OFFLOADING DECISION}

Below, a two-stage TTODA is proposed to perform faredge-to-edge and far-edge-to-cloud offloading, according to the optimal decisions given in Section IV. Moreover, TTODA can be extended to also consider memory constraints by following the approach in [10].

\section{A. Far-Edge-to-Edge Offloading (FEEO)}

During the far-edge-to-edge offloading (FEEO) process, the optimal offloading decision is found by replacing (15) into (16) for each $n$. Then, (18) is calculated in terms of $\mathcal{X}$. FEEO calculates the optimal far-edge-to-edge offloading decision, and performs the offloading of the pod that minimizes the utility function, only if there are available CPU resources in the edge server. FEEO is given in Algorithm 1.

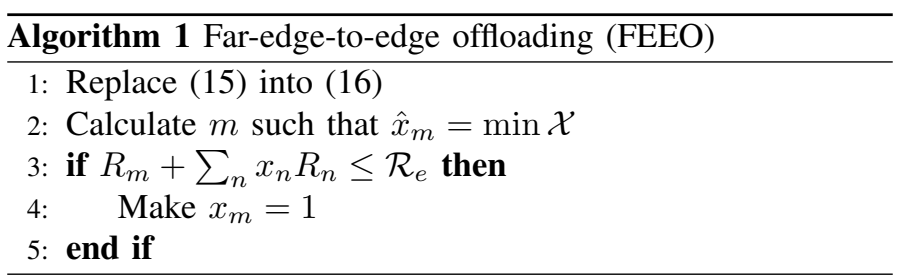

\section{B. Far-Edge-to-Cloud Offloading (FECO)}

Once the offloading of pods from the far-edge to the edge has been optimized, a far-edge-to-cloud (FECO) procedure is performed next. During FECO, the optimal offloading decision is found by replacing (15) into (12), and then calculating $\hat{y}_{n}=\hat{z}_{n}-\hat{x}_{n}$ and $\mathcal{Y}$. Next, (19) is calculated in terms of $\mathcal{Y}$. FECO performs the far-edge-to-cloud offloading of the pod that minimizes the utility function, only if $\mathrm{CPU}$ resources at the edge are exhausted. FECO is summarized in Algorithm 2. 


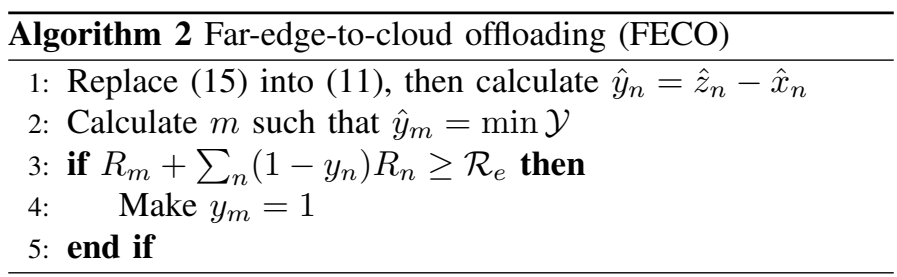

\section{Three-Tier Offloading Decision Algorithm (TTODA)}

TTODA is applied when the far-edge and edger servers are unable to handle the CPU requirements of all pods. During the first stage of TTODA, FEEO is applied to optimize the offloading of pods from the far-edge to the edge; FEEO is performed iteratively, while computational requirements of all pods cannot be met by the far-edge server, and while there are available CPU resources at the edge tier. During the second stage of TTODA, FECO is performed to optimize the offloading of pods from the far-edge to the cloud. FECO is performed iteratively, while computational requirements of all pods cannot be met by the far-edge or the edge servers. TTODA is summarized in Algorithm 3.

\begin{tabular}{l}
\hline $\begin{array}{l}\text { Algorithm } \mathbf{3} \text { Three-tier offloading } \\
\text { (TTODA) }\end{array}$ \\
\hline 1: Set $x_{n}=0, y_{n}=0, \forall n$ \\
2: Compute $\mathcal{R}_{f}, \mathcal{R}_{e}$ \\
3: while $\sum_{n}\left(1-x_{n}\right) R_{n}>\mathcal{R}_{f}$ do \\
4: Apply Algorithm 1 \\
5: end while \\
6: while $\sum_{n} x_{n} R_{n}>\mathcal{R}_{e}$ do \\
7: Apply Algorithm 2 \\
8: end while
\end{tabular}

\section{Computational Complexity of Algorithm 3 (TTODA)}

Algorithm 1 and Algorithm 2 perform one single computation each per offloaded pod. In the worst case scenario, all pods are offloaded from edge to cloud. Therefore, it is trivial that the computational complexity of Algorithm 3 is linear with the number of pods $N$, i.e. it is of order $\mathcal{O}(N)$.

\section{E. Dynamic offloading based on TTODA}

Dynamic offloading based on TTODA can be achieved by defining time slots of suitable length, and performing TTODA during each time slot. Depending on the nature of each use case, the set of variables $\mathcal{R}_{f}, \mathcal{R}_{e}$ and $R_{n}$ can be set to the instantaneous values of such parameters, or to a relevant metric such as their average, statistical expectation, etc.

\section{Numerical RESUlts}

In this Section, the performance of TTODA is evaluated for the road-safety use case of the H2020 Pledger project [13]. In order to guarantee the statistical significance of the results, Monte Carlo simulations with $10^{4}$ repetitions have been used. Network parameters in the simulations are based on worst-case measurements on our physical set-up. The far-edge-to-edge and

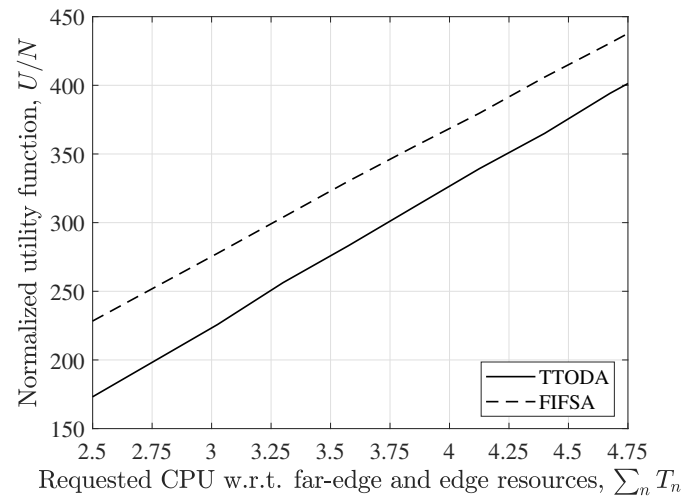

Fig. 2. Normalized utility function vs. requested CPU resources with respect to available resources at the far-edge and edge for $N=100$.

far-edge-to-cloud latencies are set to $l_{n}=35 \mathrm{~ms}$ and $l_{n}=$ $30 \mathrm{~ms}, \forall n$, respectively. Pre-loading times required by invividual pod images, $p_{n}$, are modeled as uniformly distributed random variables (UDRVs), with values in the range 10s-100s. The required pod instantiation time after pre-loading, $i_{n}$, is modeled as a UDRV with values in the range $200 \mathrm{~ms}-5 \mathrm{~s}$.

In the use case under consideration, there exists a toppriority vehicular-to-everything (V2X) stack comprised by several applications, that enables $802.11 \mathrm{p}$ communication and should be running at the far-edge node while possible. There also is a set of high-priority road hazard signalling (RHS) applications related to driver awareness situations, as defined in [16], as well as some lower priority, non-RHS applications. An integer variable $\rho_{n}$ is used to classify pods according to their priority level. Specifically, $\rho_{n}$ that takes values from the range $I_{\rho}=[1,3], \forall n$, where a value of one denotes the lowest priority value, and a value of three denotes the highest priority value. $\omega_{n}$ is then calculated as $\omega_{n}=\rho_{n} /\left(\max I_{\rho}-\min I_{\rho}\right)$.

CPU resources available at the far-edge and edge server are modeled as dimensionless units, and the normalized CPU resource requirement of pod $n, R_{n}$, is given by a uniformly distributed random variable with values in the range 0.01-0.1. Furthermore, it is assumed that servers in the cloud tier are not resource constrained, and have enough CPU resources to handle the requirements of all offloaded pods.

Below, the performance of TTODA is compared to that of a typical Kubernetes QoS model based on FIFSA [9]. Fig. 2 represents the value of the normalized utility function, defined as $U / N$, versus requested CPU resources with respect to available $\mathrm{CPU}$ resources at the edge and far-edge, i.e. $\sum_{n} T_{n}$. The number of pods is set to $N=100$, and the resources assigned to each pod increase with the computational load. TTODA outperforms the performance of FIFSA, with an average reduction of $24.45 \%$ of the value of $U / N$ achieved with FIFSA for $\sum_{n} T_{n}=2.5$. The benefit of using TTODA is larger for a lower value of $\sum_{n} T_{n}$, in which case a larger percentage of the total number of pods is allocated to the faredge and edge tiers, hence reducing the average latency and thus the value of the utility function $U$.

Fig. 3 represents the average pod response time of TTODA 


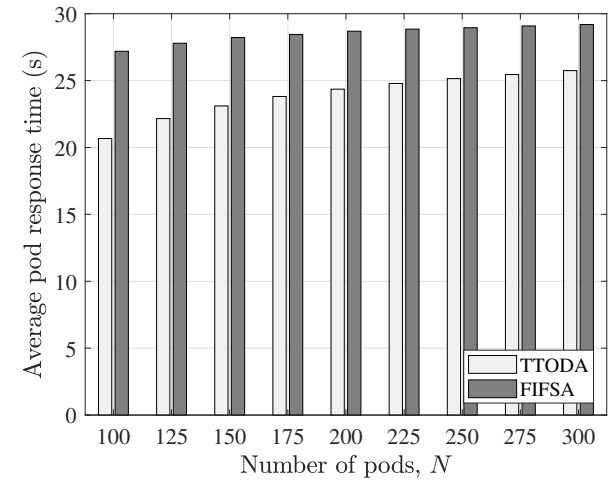

Fig. 3. Average pod response time.

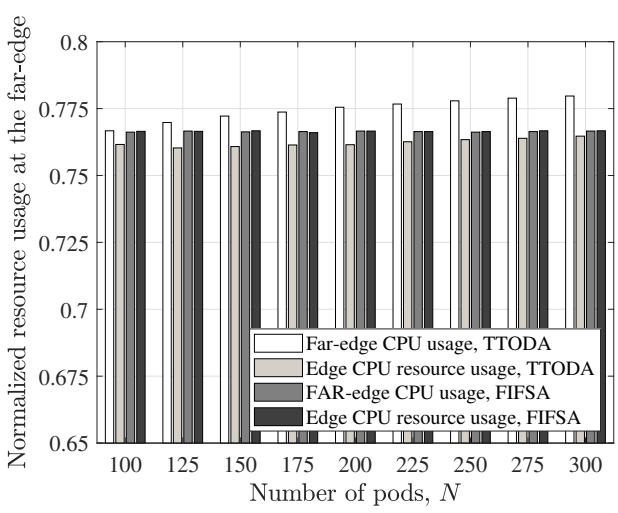

Fig. 4. Normalized resource usage at the far-edge tier.

and FIFSA, for a total CPU request of $\sum_{n} T_{n}=2.75$ and a varying number of pods. For $N=100$, TTODA improves the average pod response time by $7 \mathrm{~s}$ with respect to FIFSA, while this value is reduced to over $3 \mathrm{~s}$ for $N=300$. This result is consistent with that obtained in 2 , i.e. a larger benefit in terms of pod response time is obtained for a lower $N$, in which case the percentage of pods running on the far-edge and the edge is larger than for higher values of $N$.

Last, the normalized resource usage at the far-edge and edge servers is represented in Fig. 4, for a total CPU request of $\sum_{n} T_{n}=2.75$ and a varying number of pods, where it is assumed that a maximum CPU usage threshold of $80 \%$ has been set at both the far-edge and edge servers. As the value of $N$ increases, there is a larger diversity in the individual CPU requirements of pods, and TTODA is capable of increasing the resource usage at the far-edge server. In contrast, the server resource usage achieved with FIFSA remains approximately constant for a varying number of pods.

Overall, TTODA substantially outperforms FIFSA in terms of utility, while both algorithms have a similar computational complexity. Therefore, TTODA yields an excellent trade-off between performance and implementation. Further, TTODA can aid meeting the requirements of delay-sensitive applications, as demonstrated in Fig. 2 and 3. TTODA also increases the resource usage at the far-edge server with respect to FIFSA, further minimizing the end-to-end latency of pods, as demonstrated in Fig. 4.

\section{CONCLUSiOnS}

In this work, the problem of offloading Kubernetes tasks (or pods), was studied, from a theoretical perspective, in threetier networks. First, an utility function was formulated in terms of the cumulative weighted pod response time. Then, a utility minimization problem with CPU usage constraints was presented, and a TTODA was proposed for performing pod offloading among far-edge, edge and cloud tiers. Vertical scaling was considered, and specific hardware capabilities of each node were taken into account by feeding specific SLAs to TTODA. Numerical simulations for a road-safety use case demonstrated that TTODA outperforms a typical Kubernetes QoS model based on FIFSA in terms of utility, average pod response time and usage of far-edge resources. Moreover, TTODA yields an excellent trade-off between performance and implementation complexity. Therefore, TTODA can aid achieve reduce the end-to-end delay of latency-sensitive applications. Further, TTODA can easily be extended to scenarios with joint memory and CPU constraints.

\section{ACKNOWLEDGMENT}

This work was supported in part by the European Union's Horizon 2020 Research and Innovation Programme under grant agreement No: 871536 (PLEDGER), and by the Spanish MCIN/AEI under grant agreement no. PID2020-112675RBC43 (ONOFRE-3).

\section{REFERENCES}

[1] A. Pereira Ferreira and R. Sinnott, "A performance evaluation of containers running on managed Kubernetes services," in IEEE CloudCom, 2019, pp. 199-208.

[2] "Production-grade container orchestration," https://kubernetes.io, accessed: 2021-09-08.

[3] B. Wang et al., "A survey and taxonomy on task offloading for edge-cloud computing," IEEE Access, vol. 8, pp. 186080-186 101, 2020.

[4] F. Sun et al., "Cooperative task scheduling for computation offloading in vehicular cloud," IEEE Trans. Veh. Technol., vol. 67, no. 11, pp. 11049 $11061,2018$.

[5] N. Cheng et al., "Space/aerial-assisted computing offloading for IoT applications: A learning-based approach," IEEE J. Sel. Areas Commun., vol. 37, no. 5, pp. 1117-1129, 2019.

[6] Y. Wang et al., "Cooperative task offloading in three-tier mobile computing networks: An ADMM framework," IEEE Trans. Veh. Technol., vol. 68, no. 3, pp. 2763-2776, 2019.

[7] L. Li and H. Zhang, "Delay optimization strategy for service cache and task offloading in three-tier architecture mobile edge computing system," IEEE Access, vol. 8, pp. 170 211-170 224, 2020.

[8] P. L. Nguyen et al., "Modeling and minimizing latency in three-tier V2X networks," in IEEE GLOBECOM, 2020, pp. 1-6.

[9] J. Tang et al., "A container based edge offloading framework for autonomous driving," IEEE Access, vol. 8, pp. 33 713-33 726, 2020.

[10] E. Carmona Cejudo and M. S. Siddiqui, "An optimization framework for edge-to-cloud offloading of kubernetes pods in V2X scenarios," in IEEE GLOBECOM Workshops, 2021, pp. 1-6.

[11] “Overview of Docker Compose," https://docs.docker.com/compose/, accessed: 2021-12-10.

[12] S. Boyd and L. Vandenberghe, Convex optimization. Cambridge University Press, 2004.

[13] "Pledger project," http://pledger-project.eu/, accessed: 2021-10-20.

[14] "The most widely deployed open source cloud software in the world," https://www.openstack.org, accessed: 2021-09-12.

[15] PLEDGER, D3.2 Edge/Cloud orchestration tools I, Jun.

[16] ETSI, 3GPP TS 22.185 version 14.3.0 Release 14. LTE; Service requirements for V2X services, 2017. 